\title{
Elastografía en tiempo real (supersonic): comportamiento de la prueba en sujetos sanos de Bogotá
}

\section{Behavior of Real Time Supersonic Shear Wave Elastography in Healthy Subjects in Bogota}

\author{
Jhon E. Prieto 0., ${ }^{1}$ Santiago Sánchez P., ${ }^{2}$ Robin G. Prieto 0., ${ }^{1}$ Nathaly Garzón-Orjuela, ${ }^{3}$ Javier Eslava-Schmalbach. ${ }^{3}$
}

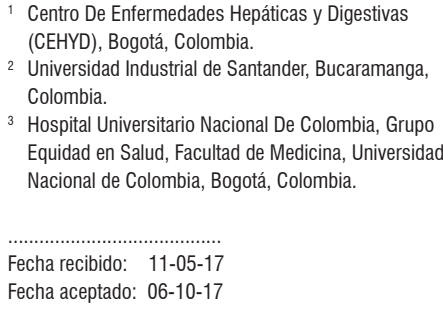

2 Universidad Industrial de Santander, Bucaramanga, Colombia.

3 Hospital Universitario Nacional De Colombia, Grupo Equidad en Salud, Facultad de Medicina, Universidad Nacional de Colombia, Bogotá, Colombia.

Fecha recibido: $11-05-17$ Fecha aceptado: 06-10-17

\begin{abstract}
Resumen
Introducción: La elastografía en tiempo real (supersonic) es una prueba no invasiva que se utiliza para determinar la elasticidad del hígado y de esa forma calcular el grado de fibrosis hepática; en algunas ocasiones, reemplaza la toma de la biopsia hepática y es útil para el seguimiento de la mayoría de pacientes con hepatopatías. En Colombia, la prueba se introdujo recientemente y no existen hasta el momento estudios del comportamiento de la prueba en personas sanas. Objetivo: evaluar el comportamiento de la elastografía en tiempo real en sujetos sanos en Bogotá. Materiales y métodos: estudio piloto retrospectivo de sujetos sanos entre 15 y 37 años, entre el 1 de junio de 2016 y el 31 de marzo del 2017. Se realizaron la historia clínica y evaluación básica de la función hepática para descartar anormalidades. Resultados: la serie incluyó 33 sujetos sanos, $60,6 \%$ mujeres y $39,4 \%$ hombres con una edad promedio de 24,8 años. Al practicar la prueba se encontró un valor promedio de 4,28 kilopascales ( $\mathrm{kPa})$, con una diferencia mínima entre sexos, siendo mayor la rigidez hepática en los hombres. Conclusiones: los valores promedio de la rigidez hepática en 33 sujetos sanos en Bogotá son los mismos reportados en los estudios iniciales de las pruebas a nivel internacional; estos hallazgos sugieren que la prueba puede ser muy útil en nuestro medio para la evaluación de pacientes con hepatopatías.
\end{abstract}

\section{Palabras clave}

Elastografía en tiempo real; supersonic; fibrosis hepática; adultos sanos.

\begin{abstract}
Introduction: Real-time supersonic shear wave elastography (SSI) is a noninvasive test that is used to determine the elasticity of the liver and thus calculate the degree of liver fibrosis. Sometimes, it can replace a liver biopsy, and it is useful for monitoring the majority of patients with liver disease. In Colombia, the test has recently been introduced, so there have been no studies of test in healthy people here until now. Objective: The objective of this study was to evaluate the behavior of the elastography in real time in healthy subjects in Bogotá. Materials and methods: This is a retrospective pilot study of healthy subjects between 15 and 37 years old who underwent SSI between June 1, 2016 and March 31, 2017. A clinical history was taken and basic evaluation of liver function was performed to rule out abnormalities. Results: The series included 33 healthy subjects, $60.6 \%$ women and $39.4 \%$ men with an average age of 24.8 years. When the test was performed, an average value of 4.28 kilopascals (kPs) was found, with minimal difference between the sexes but greater hepatic rigidity in men. Conclusions: The mean values of hepatic rigidity in 33 healthy subjects in Bogotá are the same as those reported in the initial studies internationally. These findings suggest that the test may be very useful for the evaluation of patients with liver disease in our environment.
\end{abstract}

\section{Keywords}

Real-time elastography; supersonic; hepatic fibrosis; healthy adults. 


\section{INTRODUCCIÓN}

Las enfermedades hepáticas crónicas desarrollan paulatinamente diferentes grados de fibrosis que pueden culminar en la cirrosis. Determinar en cuál etapa de la enfermedad se encuentran nuestros pacientes ha sido de interés permanente, puesto que esto determina la necesidad de intervención temprana, como en el caso de la hepatitis C y B (1-3), $\mathrm{y}$ adicionalmente genera un pronóstico en estas y otras hepatopatías. Clásicamente, la determinación del grado de fibrosis se establece con la biopsia hepática; sin embargo, los riesgos por un procedimiento invasivo, los costos y en ocasiones la no aceptación por parte del paciente han hecho emerger a los marcadores no invasivos de fibrosis hepática como una alternativa (4-9).

La elastografía en tiempo real o supersonic (elastography "Shear Wave ${ }^{\mathrm{Tu} \text { ", }}, \mathrm{SWE}^{\mathrm{rux}}$ ) es una técnica de elastografía basada en ultrasonido que tiene la capacidad de cartografiar y medir la rigidez hepática (10). Se ha implementado en un sistema de ultrasonido el Aixplorer, que proporciona una imagen bidimensional, cuantitativa y de color de la rigidez del tejido hepático en tiempo real. El supersonic es una prueba no invasiva que determina la elasticidad del hígado y calcula el grado de fibrosis hepática; en algunas ocasiones, reemplaza la toma de la biopsia hepática y es útil para el seguimiento de la mayoría de pacientes con hepatopatías (11-14).

En Colombia, la prueba se introdujo recientemente y no existe hasta el momento ningún estudio del comportamiento de la prueba en nuestro medio, por lo cual el objetivo de este estudio fue evaluar el comportamiento de la elastografía en tiempo real en sujetos sanos en un centro de atención de enfermedades hepáticas en Bogotá.

\section{MATERIALES Y MÉTODOS}

Se realizó un estudio piloto retrospectivo en sujetos sanos voluntarios entre 15 y 37 años, atendidos entre el 1 de junio de 2016 y el 31 de marzo del 2017 en un centro de la ciudad de Bogotá, con consentimiento verbal de los sujetos incluidos. Se obtuvo información de la historia clínica completa con examen físico y evaluación básica de la función hepática para descartar anormalidades. Los criterios de inclusión se basaron en la consideración de individuo sano:

- La historia clínica descartaba cualquier antecedente de importancia para hepatopatía (historia de hepatitis, trasfusiones, drogadicción, obesidad, ingesta de alcohol, entre otros).

- El examen físico se consideró normal: sin estigmas de hepatopatía crónica y el hígado era normal a la palpación.

- El índice de masa corporal (IMC) era normal, es decir, entre 18 y $25 \mathrm{~kg} / \mathrm{m}^{2}$.
- Los paraclínicos básicos se encontraban en niveles normales:

- Transaminasas $<30$ unidades internacionales (UI)/dL

- Niveles de índice internacional normalizado (INR) entre $0,8-1,2$

- Bilirrubinas entre 0,3 y $1,3 \mathrm{mg} / \mathrm{dL}$

- Albúmina entre 3,5 y 5,0 g/dL

$\mathrm{Al}$ ser incluidos en el estudio, a todos los participantes se les practicó la prueba de elastografía mediante el supersonic siguiendo el protocolo recomendado por el fabricante y utilizado en los estudios pivotales publicados así : la elastografía en tiempo real se realizó utilizando el sistema de ultrasonido Aixplorer (SuperSonic Imagine S. A. Aix-en-Provence, Francia) con una sonda convexa de banda ancha (SC6-1). En el modo SWE, se crean ondas de cizalladura o corte en el tejido a partir de la fuerza de radiación acústica generada por impulsos dados por el ultrasonido. La velocidad de la onda de corte se estima mediante una adquisición tipo Doppler (10). La velocidad de onda de corte se usa para calcular la rigidez de los tejidos mediante la fórmula $\mathrm{E}=\mathrm{pc}^{2}$; $E$ es la elasticidad del tejido en $\mathrm{kPa}, p$ es la densidad del tejido $\left(\mathrm{kg} / \mathrm{m}^{3}\right)$ y $c$ es la velocidad de la onda cortante $(\mathrm{m} / \mathrm{s})$.

Los datos de elasticidad se codifican con colores mediante el software creando un mapa bidimensional de rigidez tisular, que se muestra en un recuadro o caja sobre la imagen en modo B convencional. Se establece una región de interés (ROI) en el recuadro donde se realiza la medida de la rigidez hepática; utilizamos un promedio de $2 \mathrm{~cm}$ de ROI y un recuadro de $3,5 \times 2,5 \mathrm{~cm}$. Los participantes asistieron en ayunas y las mediciones se realizaron en el lóbulo derecho del hígado, a través de los espacios intercostales, con el sujeto acostado en posición supina con el brazo derecho en la abducción máxima. Las mediciones se clasificaron como fallidas cuando no se obtuvo señal en el recuadro o esta era mínima en todas las adquisiciones. Los análisis estadísticos se basaron en los datos obtenidos de 5 mediciones consecutivas, debido a que la imagen en el recuadro es el resultado de aproximadamente 3 imágenes promediadas en el tiempo; al tomar datos de 5 mediciones, realmente se están haciendo 15 mediciones.

La información se analizó de manera descriptiva mediante el cálculo de medidas de tendencia central y de dispersión para las variables cuantitativas, y las variables categóricas se resumieron en frecuencias absolutas y relativas. Se validó el supuesto de normalidad de los datos continuos mediante la prueba de Shapiro-Wilk. La medición de la diferencia entre el grupo de hombres y mujeres se realizó mediante la prueba de comparación de medias ( $T$ test), y para más de 2 grupos, con one-way analysis of variance (ANOVA). Todos los análisis se realizaron con el paquete estadístico STATA versión 13. 


\section{RESULTADOS}

Durante el período de estudio se incluyeron 33 sujetos sanos, cuyas características generales y clínicas se detallan en la Tabla 1. La edad promedio fue de 24,8 años, el IMC sugiere pacientes delgados con todos los paraclínicos completamente normales.

Tabla 1. Datos generales y paraclínicos de los pacientes

\begin{tabular}{lccc}
\hline \multicolumn{1}{c}{ Variable } & $\begin{array}{c}\text { Mujeres } \\
(\mathbf{n}=20) \\
\text { Media (DE) }\end{array}$ & $\begin{array}{c}\text { Hombres } \\
(\mathbf{n}=13) \\
\text { Media (DE) }\end{array}$ & $\begin{array}{c}\text { Total }(\mathbf{n}=33) \\
\text { Media (DE) }\end{array}$ \\
\hline Edad en años & $27,1(6,7)$ & $20(19-23)^{*}$ & $24,8(6,3)$ \\
IMC & $20,9(1,8)$ & $19,6(2,1)$ & $20,4(2,01)$ \\
Glóbulos blancos (cel/mL) & $6301(1204)$ & $6160(1437)$ & $6246(1281)$ \\
Hemoglobina (g/dL) & $14,1(0,8)$ & $16,4(0,8)$ & $15,06(1,4)$ \\
Hematócrito (\%) & $42(41-44)^{*}$ & $49(47-50)^{*}$ & $44(42-49)^{*}$ \\
Plaquetas (cel/mm ${ }^{3)}$ & 282700 & 270846 & 278030 \\
& $(50627)$ & $(37799)$ & $(45741)$ \\
Glicemia (mg/dL) & $84,3(4,7)$ & $84(81-88)^{*}$ & $85(81-88)^{*}$ \\
AST (UI/dL) & $18(17-20)^{*}$ & $18,3(2,0)$ & $18(17-20)^{*}$ \\
ALT (UI/dL) & $18,1(7,1)$ & $21,7(5,6)$ & $19,5(6,7)$ \\
GGT (UI/dL) & $22(13-35)^{*}$ & $23(19-56)^{*}$ & $22(16-40)^{*}$ \\
FA (UI/dL) & $71(59-90)^{*}$ & $107,5(22,0)$ & $89,1(29,9)$ \\
Bilirrubina total (mg/dL) & $0,5(0,3-0,8)^{*}$ & $0,9(0,46)$ & $0,5(0,4-1)^{*}$ \\
Bilirrubina directa & $0,2(0,1-0,3)^{*}$ & $0,36(0,22)$ & 0,2 \\
(mg/dL) & & & $(0,1-0,3)^{*}$ \\
Bilirrubina indirecta & $0,3(0,2-0,4)^{*}$ & $0,5(0,3-0,9)^{*}$ & 0,3 \\
(mg/dL) & & & $(0,2-0,5)^{*}$ \\
Proteínas totales (g/dL) & $7,51(0,39)$ & $7,6(0,34)$ & $7,54(0,37)$ \\
Albúmina (g/dL) & $4,5(0,37)$ & $4,6(0,48)$ & $4,54(0,41)$ \\
\hline
\end{tabular}

*Mediana (rango intercuartílico [RIQ])-prueba de normalidad ShapiroWilk. AST: aspartato aminotransferasa; ALT: alanina transaminasa; Cel: células; DE: desviación estándar; FA: fosfatasa alcalina; GGT: gammaglutamil transferasa.

Todos los 33 pacientes presentaron un examen físico normal, no ingerían alcohol, los estudios para hepatitis B y $C$ fueron negativos y la ecografía abdominal era normal.

Los valores de rigidez hepática en los 33 sujetos muestran una media de 4,28 $\mathrm{kPa}$ con una desviación estándar (DE) de 0,61. Al analizar por sexo, se encontró una diferencia significativa entre hombres y mujeres en las medias de los valores de rigidez hepática $(\mathrm{p}=0,0048)$ (Figura 1 ). Ninguna de las pruebas se consideró fallida. La elastografía en tiempo real duró entre 10 y 15 minutos por paciente.

En la Tabla 2 se evidencian los valores de rigidez hepática por grupo etario; de los pacientes analizados, el grupo de 21 a 26 años fue el que presentó un valor mayor de rigidez.

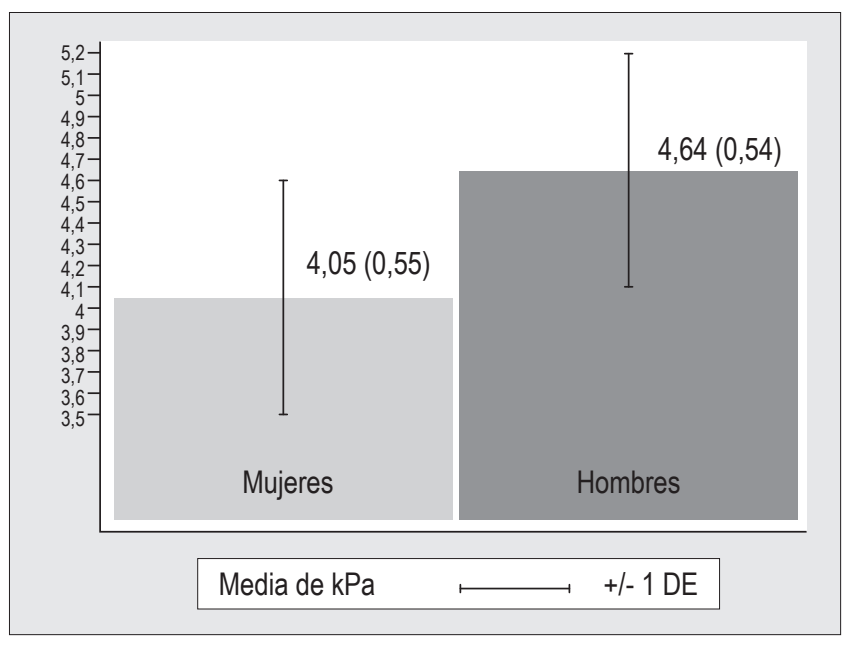

Figura 1. Valores de la rigidez hepática según sexo. DE: desviación estándar; $\mathrm{kPa}$ : valores de kilopascales.

Tabla 2. Valores de rigidez hepática por grupo etario (distribución por cuartiles)

\begin{tabular}{ccc}
\hline Grupo etario & $\mathbf{N}$ & $\begin{array}{c}\text { Media (DE) } \\
\text { valores en kPa* }\end{array}$ \\
\hline $15-20$ & 12 & $4,3(0,68)$ \\
$21-26$ & 7 & $4,42(0,40)$ \\
$27-30$ & 9 & $4,04(0,62)$ \\
$31-37$ & 5 & $4,3(0,73)$ \\
$15-37$ & 33 & $4,28(0,61)$ \\
\hline
\end{tabular}

${ }^{*}$ No hay diferencias significativas entre los grupos de edad (one-way ANOVA). DE: desviación estándar; kPa: valores de kilopascales.

\section{DISCUSIÓN}

A pesar de ser un estudio piloto con una muestra de 33 pacientes, los hallazgos son de gran relevancia, debido a que en la mayoría de estudios de elastografía en tiempo real no se define con certeza el valor de la normalidad. En el metaanálisis de Changtian Li (15), en el que se incluyeron 8 estudios con 934 pacientes, la mayoría con hepatitis $\mathrm{Co}$ $\mathrm{B}$, el punto de corte para fibrosis significativa F2 o mayor varió entre 6,6 y $8,8 \mathrm{kPa}$. Ferraioli (16) encontró un punto de corte de 7,1 kPa, con valor promedio 6,2 $\mathrm{kPa}$ para F0 y F1 con un RIQ entre 5,1-6,8 y más centrado en sus análisis en fibrosis significativa.

Dentro de los estudios que buscan el valor normal, Cha (17), en un estudio retrospectivo con 97 pacientes sin enfermedad hepática, encontró un valor promedio de rigidez hepática de 5,4 kPa para definir normalidad; el punto de corte óptimo para discriminar el hígado no enfermo del enfermo crónico fue de $6,9 \mathrm{kPa}$, con una sensibilidad del $94 \%$. Sirli (18) evaluó la rigidez hepática de 82 pacientes 
sin patología hepática conocida (70 con medidas confiables), con edades entre 18 y 76 años. El valor medio de la rigidez determinada por supersonic fue de $6 \pm 1,4 \mathrm{kPa}$. Los valores para los hombres fueron significativamente mayores que para las mujeres. Este estudio incluyó pacientes de todas las edades, algunos con sobrepeso y obesidad, y se encontraron valores normales de hasta $9 \mathrm{kPa}$ que, en otros estudios, están en el rango de F2. Con respecto a los 33 sujetos analizados, se observó una diferencia entre el grupo de hombres y mujeres en los valores de rigidez hepática; estos mismos resultados se observaron en otros estudios.

En el estudio de Suh y colaboradores (19), con 196 donantes potenciales para trasplante de hígado con donante vivo, el rango de referencia para la elasticidad hepática normal confirmada por biopsia fue 2,6-6,2 $\mathrm{kPa}$, con una elasticidad media de 4,4 kPa. El valor medio de elasticidad hepática normal en nuestro estudio fue 4,28 $\mathrm{kPa}$, que está en concordancia con los datos de Suh (en cuyo estudio la mayoría eran sujetos delgados similares a los nuestros) y con valores obtenidos en voluntarios sanos sin corroboración histológica $(4,6-4,9 \mathrm{kPa})(20)$.

Somos conscientes de las limitaciones de nuestro estudio:

- El tamaño de la muestra es pequeño, pero como estudio piloto quisimos únicamente presentar un reporte preliminar de la rigidez hepática normal en nuestros pacientes, con la intención de ampliar la muestra en estudios futuros.

- Nuestros pacientes no tuvieron biopsias hepáticas como prueba de oro. El concepto de normalidad se basó en criterios clínicos y de laboratorio, pero tomar biopsias hepáticas a sujetos sanos no está justificado éticamente.

- Hubo un número desigual de pacientes hombres y mujeres, lo que podría explicar en parte la diferencia presentada, pero esto seguramente se solucionará en próximos estudios.

\section{CONCLUSIONES}

El valor promedio de la rigidez hepática en 33 sujetos sanos en Bogotá es similar a los reportados en los estudios iniciales de las pruebas a nivel internacional. Estos hallazgos sugieren que la prueba puede ser muy útil en nuestro medio para la evaluación de pacientes con hepatopatías. Reconocemos las debilidades metodológicas del estudio; sin embargo, como estudio piloto es el punto de partida para la investigación de este tema en nuestro medio.

\section{Agradecimientos}

Agradecemos a nuestros pacientes por habernos permitido ser nuestro objeto de estudio.

\section{Conflicto de intereses}

Los autores declaran no tener ningún conflicto de intereses.

\section{Fuentes de financiación}

Los autores declaran no tener ninguna fuente de financiación para la realización del estudio.

\section{REFERENCIAS}

1. Ghany MG, Strader DB, Thomas DL, et al. Diagnosis, management, and treatment of hepatitis C: an update. Hepatology. 2009;49(4):1335-74. https://doi.org/10.1002/hep.22759

2. European Association for the Study of the Liver. EASL Recommendations on Treatment of Hepatitis C 2016. J Hepatol. 2017;66(1):153-94.

3. Sundaram V, Kowdley K. Management of chronic hepatitis B infection. BMJ. 2015;351:h4263.

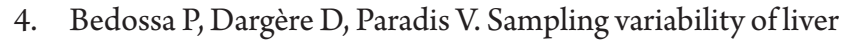
fibrosisin chronichepatitis C. Hepatology.2003;38(6):144957. https://doi.org/10.1053/jhep.2003.09022 https://doi. org/10.1016/j.hep.2003.09.022 https://doi.org/10.1016/ S0270-9139(03)80411-1

5. Poynard T, Munteanu M, Imbert-Bismut F, et al. Prospective analysis of discordant results between biochemical markers and biopsy in patients with chronic hepatitis C. Clin Chem. 2004;50(8):1344-55. https://doi.org/10.1373/ clinchem.2004.032227

6. Bravo AA, Sheth SG, Chopra S. Liver biopsy. N Engl J Med. 2001;344(7):495-500. https://doi.org/10.1056/ NEJM200102153440706

7. Cassinotto C, Lapuyade B, Mouries A, et al. Non-invasive assessment of liver fibrosis with impulse elastography: comparison of Supersonic Shear Imaging with ARFI and FibroScan. J Hepatol. 2014;61(3):550-7. https://doi. org/10.1016/j.jhep.2014.04.044

8. Castera L. Invasive and non-invasive methods for the assessment of fibrosis and disease progression in chronic liver disease. Best Pract Res Clin Gastroenterol. 2011;25(2):291303. https://doi.org/10.1016/j.bpg.2011.02.003

9. European Association for Study of Liver; Asociacion Latinoamericana para el Estudio del Higado. EASLALEH Clinical Practice Guidelines: Non-invasive tests for evaluation of liver disease severity and prognosis. J Hepatol. 2015;63(1):237-64. https://doi.org/10.1016/j. jhep.2015.04.006

10. Muller M, Gennisson JL, Deffieux T, et al. Quantitative viscoelasticity mapping of human liver using supersonic shear imaging: preliminary in vivo feasibility study. Ultrasound Med Biol. 2009;35(2):219-29. https://doi.org/10.1016/j. ultrasmedbio.2008.08.018

11. Samir AE, Dhyani M, Vij A, et al. Shear-wave elastography for the estimation of liver fibrosis in chronic liver disease: 
determining accuracy and ideal site for measurement. Radiology. 2015;274(3):888-96. https://doi.org/10.1148/ radiol.14140839

12. Tada T, Kumada T, Toyoda H, et al. Utility of real-time shear wave elastography for assessing liver fibrosis in patients with chronic hepatitis $\mathrm{C}$ infection without cirrhosis: Comparison of liver fibrosis indices. Hepatol Res. 2015;45(10):E122-9. https://doi.org/10.1111/hepr.12476

13. Yoneda M, Thomas E, Sclair SN, et al. Supersonic Shear Imaging and Transient Elastography With the XL Probe Accurately Detect Fibrosis in Overweight or Obese Patients With Chronic Liver Disease. Clin Gastroenterol Hepatol. 2015;13(8):1502-9.e5. https://doi.org/10.1016/j. cgh.2015.03.014

14. Procopet B, Berzigotti A, Abraldes JG, et al. Real-time shear-wave elastography: applicability, reliability and accuracy for clinically significant portal hypertension. J Hepatol. 2015;62(5):1068-75. https://doi.org/10.1016/j. jhep.2014.12.007

15. Li C, Zhang C, Li J, et al. Diagnostic Accuracy of Real-Time Shear Wave Elastography for Staging of Liver Fibrosis: A
Meta-Analysis. Med Sci Monit. 2016;22:1349-59. https:// doi.org/10.12659/MSM.895662

16. Ferraioli G, Tinelli C, Dal Bello B, et al. Accuracy of real-time shear wave elastography for assessing liver fibrosis in chronic hepatitis C: a pilot study. Hepatology. 2012;56(6):2125-33. https://doi.org/10.1002/hep.25936

17. Cha SW, Jeong WK, Kim Y, et al. Nondiseased liver stiffness measured by shear wave elastography: a pilot study. J Ultrasound Med. 2014;33(1):53-60.

18. Sirli R, Bota S, Sporea I, et al. Liver stiffness measurements by means of supersonic shear imaging in patients without known liver pathology. Ultrasound Med Biol. 2013;39(8):1362-7.

19. Suh CH, Kim SY, Kim KW, et al. Determination of normal hepatic elasticity by using real-time shear-wave elastography. Radiology. 2014;271(3):895-900. https://doi. org/10.1148/radiol.14131251

20. Ferraioli G, Tinelli C, Zicchetti M, et al. Reproducibility of real-time shear wave elastography in the evaluation of liver elasticity. Eur J Radiol. 2012;81(11):3102-6. https://doi. org/10.1016/j.ejrad.2012.05.030 